\title{
A Mobile Video Surveillance System based SL4A and HTTP and Scripting Languages
}

\author{
Huajun Lei , Yiliang Xing* \\ Software Engineering Department, Hainan College of Software Technology ,Qionghai 571400, \\ China \\ *Corresponding author's e-mail: xylpaper@163.com
}

Keywords: SL4A, android, video surveillance, HTTP, php.

\begin{abstract}
It is of great significance to extend the android SL4A video camera service to pc desktop and mobile phone applications for the scripting languages, such as JavaScript and PHP. In this paper, we propose a method based on SL4A, script language and HTTP communication protocol to expand SL4A video service, and design and implement a mobile video surveillance system. Firstly, the system is designed as a system composed of mobile phone camera monitoring server and web client, and then the camera image data of the mobile phone camera monitoring server is collected by SL4A, and then the image data is transmitted according to the HTTP protocol, and lastly the system is developed by PHP scripting language. The experimental results show that the method can expand the mobile video camera service to pc and other mobile phone applications, and has the advantages of simple implementation, easy integration and cross-language.
\end{abstract}

\section{Introduction}

The replacement of smart phones is very fast, which led to the high elimination frequency of old mobile phone. Smart phones are usually equipped with high-definition camera. If mobile phone camera can be integrated into the pc or mobile phone applications, then old phone will continue to play an important role, it will be a very meaningful thing.

On the old mobile phone camera re-use, there are two kinds of common methods, one method is to use the third party software[1], such as IP Webcam and DroidCam, to display mobile phone camera video ,and the other is to use Java language to develop new features about camera [2][3]. The method of using the third party software to reconstruct the old mobile phone camera has the advantages of simple realization, supporting the original data of the sensor, good compatibility with the client and high image quality, but it has disadvantages of mobile phone camera image data and processed sensor data integration difficulty and high cost, and it's difficult for developers to pull together processed geographic sensor data and image data. The advantage of using Java language to develop application for old mobile phone camera is that the function is flexible and a strong real-time is perfect. However, because Java language, video coding and other complex technologies need to be mastered, so there are some problems such as slow progress and high cost.

We present the method of the paper to design and develop a mobile phone camera application, which is based on SL4A to collect images of the mobile phone, transfer images with the HTTP communication protocol, and use PHP script language to develop the application.

\section{SL4A Framework, Script Technology and HTTP Protocol}

\subsection{SL4A Framework Working Principle}

SL4A[4][5][6][7] is an android application that the first name is ASE (Android Scripting Environment), and its function is to support the development of android applications using scripting languages, which makes android application development easier and faster.

SL4A is developed and maintained by Google Corporation employee Damon Kohler, and it supports a lot of scripting languages, including Python, Perl, JRuby, Lua, BeanShell, JavaScript, Tcl and shell, etc. 


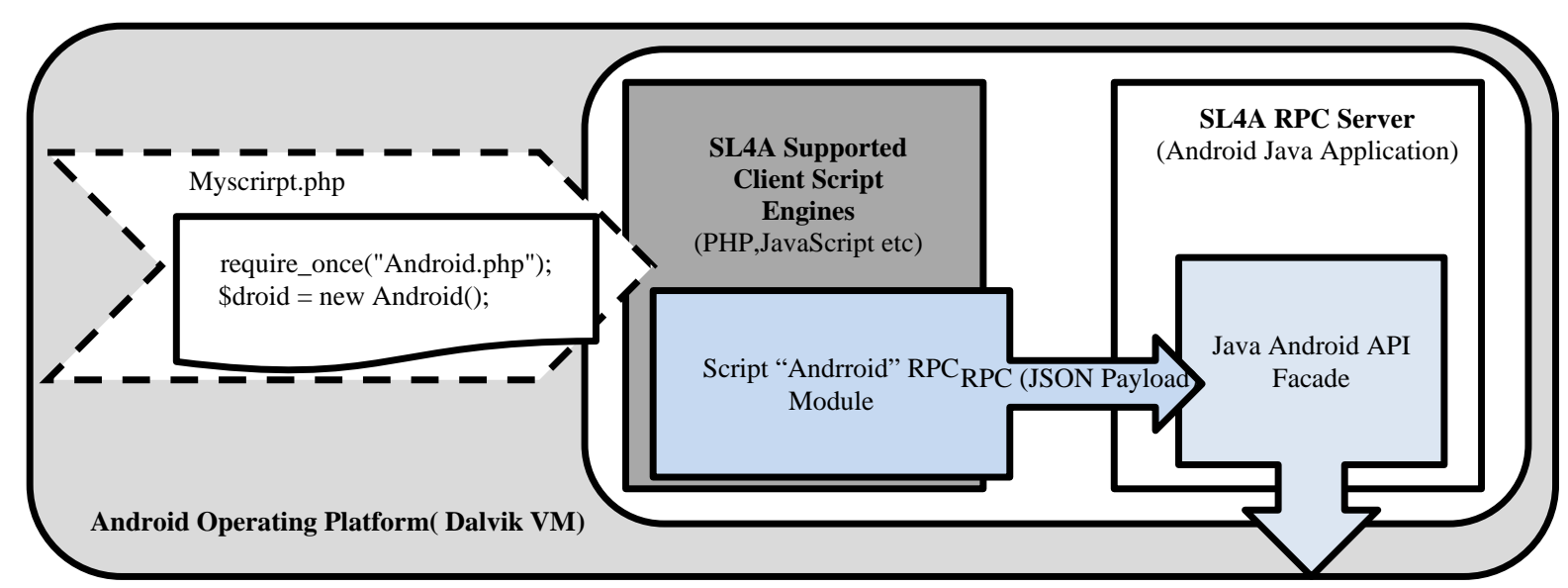

Fig. 1 SL4A architecture

SL4A implements the service mechanism of local scripting languages to access the android native services. As long as compatible with the JSON RPC communication interface provided by SL4A, any local scripting languages can access the android services provided by the SL4A methods. The SL4A architecture is shown in Fig. 1.

From Figure1, you can see that SL4A is composed of client and Server. The client is the SL4A supported client script engines, and it is responsible for parsing the local script application. As long as a local script application implementation SL4A JSON RPC communication interface, the application can easily access the android native services of the server. The server to receive SL4A RPC call from a client will call java android API to provide the android native services for the client.

The communication process of the client and the server is that the client parses android service call from the script application firstly, and the client uses SL4A JSON RPC interface to send android service request secondly, and the server invokes java android api to complete the request and retrace the call path to return executed results to the client lastly.

\subsection{SL4A Script Technology}

A company named irontec [8] launched an open source project name PFA(PHP for Android). The project can allow PHP script language to run on android platform. The PFA project also provides APK tools and documentations to support PHP running on android platform. The project provides a APK application named PhpForAndroid, the APK is the PHP engine. After the installation of the APK application, PHP script can run on the android platform. The latest version of the APK application is 0.3, which supports PHP 5.3.3, JSON, sockets and openssl. About the android PHP engine, you can visit the site http://www.phpforandroid.net/doku.php to learn more about its information.

\subsection{HTTP protocol}

A protocol is a set of rules or rules that must be observed in the communication between two computers in a network. HTTP(Hypertext Transfer Protocol) is a communication protocol that allows hypertext markup language documents to be transmitted from the web server to the client browser. The ommunication process of web server and browser is that browser client sends request to web server firstly, and web server receives the request to generate corresponding response secondly, and web server returns the response to browser client thirdly, and browser client parses the data in response and displays the content finally. Request and Response packets in communication process must conform to the HTTP protocol.

The HTTP protocol specifies that the Response packet consists of four parts. The first part is Response line, and the second part is Response header, and the third part is empty line, and the last part is Response body. Response line specifies the HTTP communication protocol version, communication status, and status description. It consists of three data domains which are HTTP/version-number,status-code , and description. The three parts are separated by spaces. HTTP/version-number represents the version number of the HTTP protocol, the version-number value is generally 1.1. Status-code is a 3 bit number, the most common value is 200 , meaning that 
web server has successfully responded to browser client request. Description is a state code description, when status-code is 200, the description value is "OK".

\section{System Design and Implementation}

\subsection{System Requirements and Design}

This paper designed and developed the system based on the server/browser software architecture design theory, and the system is composed of the mobile phone camera monitoring server and the web client, and the system architecture is shown in Fig. 2.

The mobile phone camera monitoring server runs on the phone, and its function is to collect the camera image. It is composed of script application, script engine and SL4A component. Script application calls the SL4A JSON RPC interface by the script engine to collect the camera image of the mobile phone, and transfers the camera image data based on the HTTP communication protocol. The script engine is responsible for parsing and running script applications. SL4A is responsible for responding to script application request and with the help of android native method to capture the camera image.

The function of the web client is to request, transmit and display camera images of the mobile phone camera monitoring server via HTTP protocol, and it is developed by HTML and JavaScript, which can be run on PC or mobile browsers.

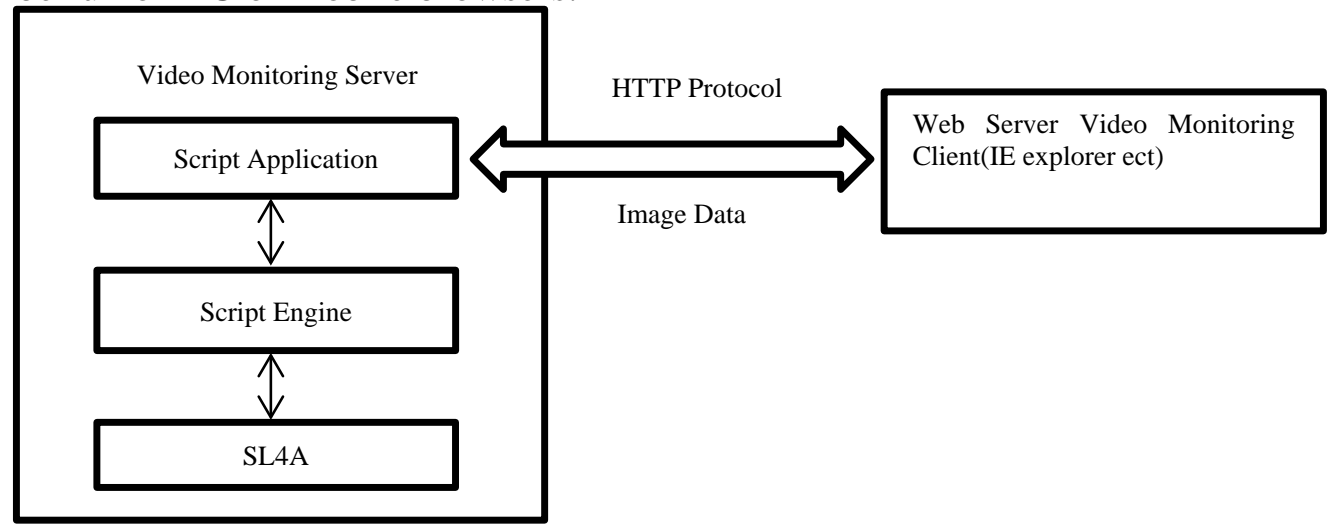

\subsection{System Implementation}

Fig. 2 the system's architecture

The code of the system is given in this paper. The code is composed of Web client file client.htm and mobile phone monitor server file server.php. The client application runs in browser, and it requests the server camera image data in asynchronous mode. The server application runs in mobile phone, and it will listen the client request, collect the camera image data and transmit the phone camera image data repeatedly in this order. The main code of the file client.htm is shown below.

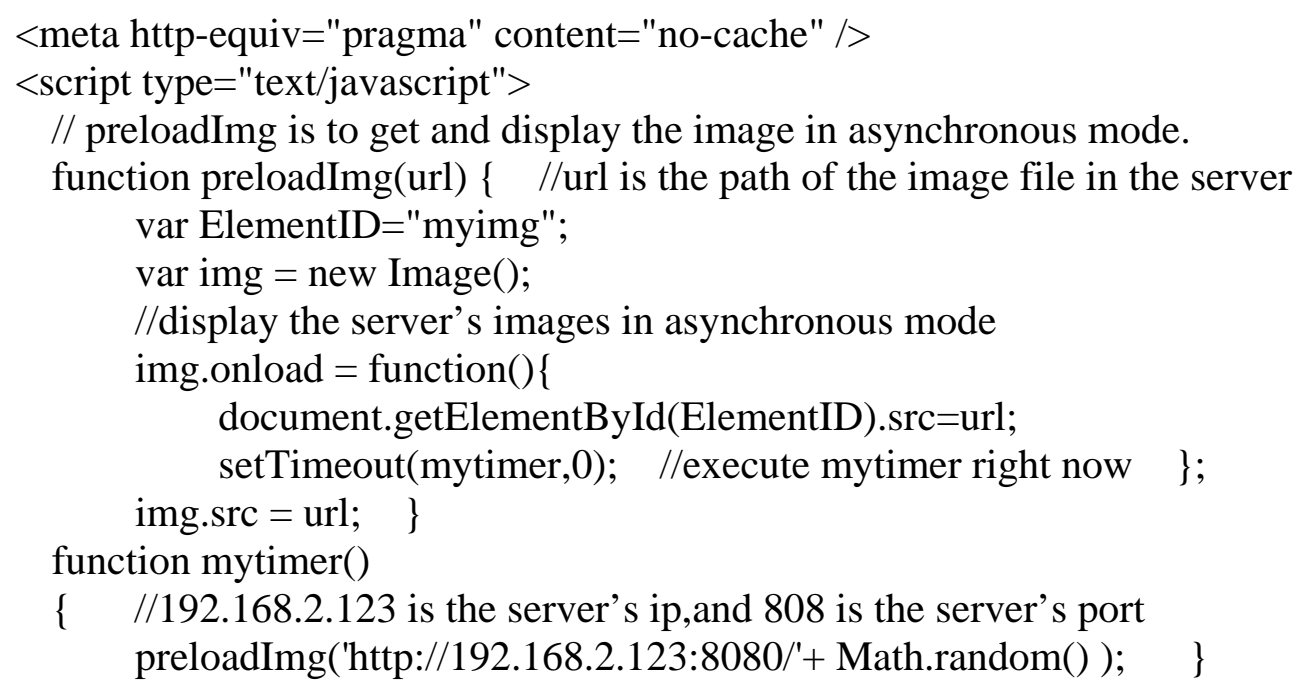


setTimeout(mytimer,10); //after 10 millisecond to execute mytimer $</$ script $>$

$<$ div $><$ img width="300" height="300" id="myimg"/> </div $>$

The main code of the file server.php is shown below.

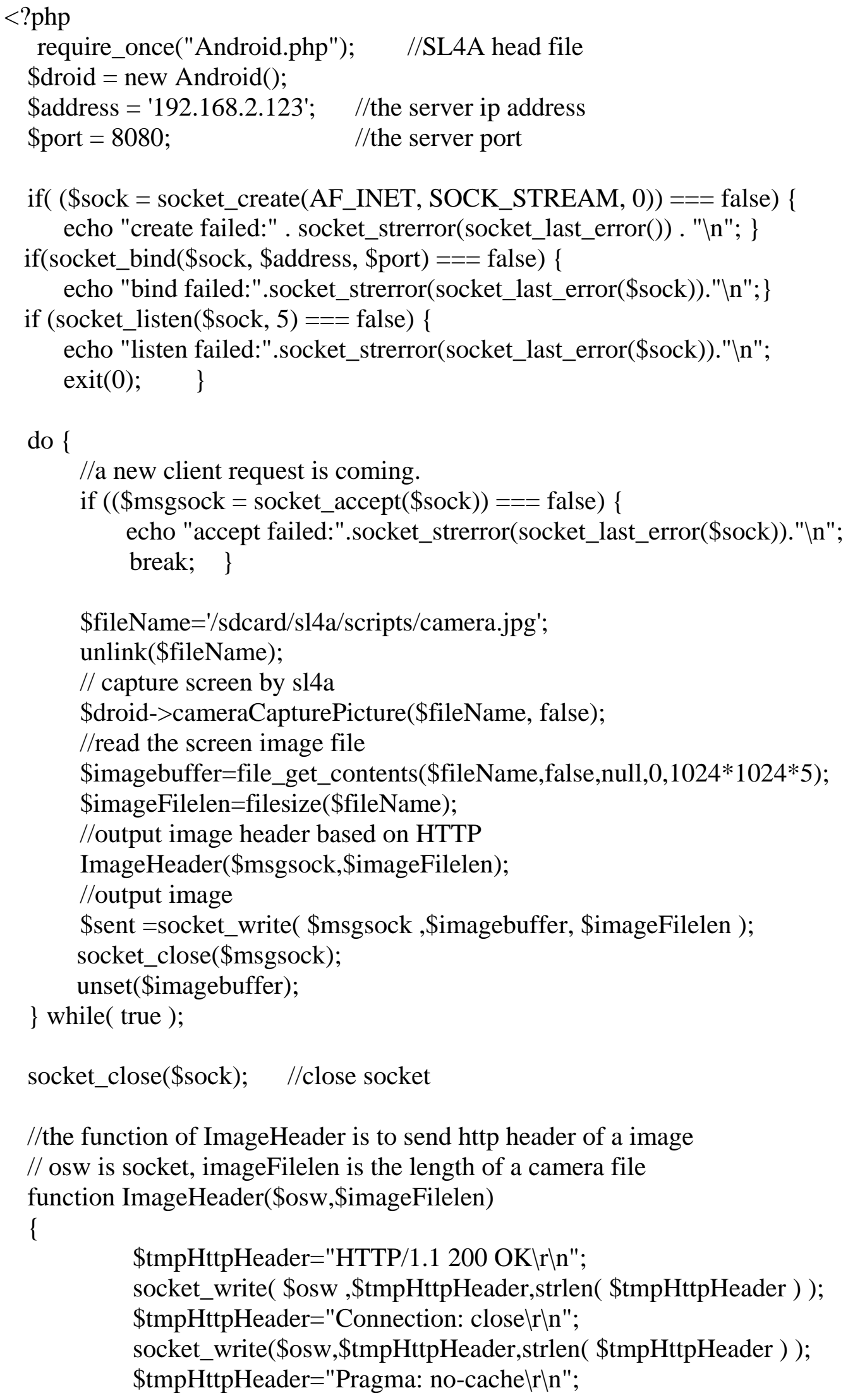

\$tmpHttpHeader="HTTP/1.1 200 OK\r\n";

socket_write( \$osw ,\$tmpHttpHeader,strlen( \$tmpHttpHeader ) );

\$tmpHttpHeader="Connection: close $\backslash$ rın";

socket_write(\$osw,\$tmpHttpHeader,strlen( \$tmpHttpHeader ) );

\$tmpHttpHeader="Pragma: no-cache $\backslash$ r|n"; 
socket_write(\$osw,\$tmpHttpHeader,strlen( \$tmpHttpHeader ) ); \$tmpHttpHeader="Content-Length: "+\$imageFilelen +"\rın"; socket_write(\$osw,\$tmpHttpHeader,strlen( \$tmpHttpHeader ) ); \$tmpHttpHeader="Content-Type: image/jpeg $\backslash r \mid n \backslash r \backslash n " ;$

socket_write(\$osw,\$tmpHttpHeader,strlen( \$tmpHttpHeader ) ); \} ?>

\section{Experiment and Analysis}

The method is passed in mobile phone named red rice 1S (mobile phone configuration: Android 4.4.2; quad core CPU 1.3Ghz ; 1 GB memory; 8 GB flash; 8 million pixels rear camera) and SL4A and PFA/RHINO and WiFi and Internal Explorer 9 browser. Mobile phone camera image's resolution is $3264 * 1840$ pixels, and its color depth is 24-bit color. It is an indoor surveillance screenshot in fig. 3, and screenshot frame number is 13, and it takes 52 seconds to shoot the 13 pictures, and it takes 194 seconds to take 50 pictures, with an average of about 3.88 seconds per frame. It is an outdoor surveillance screenshot in Fig. 4, and screenshot frame number is 4, and it takes 28 seconds to shoot the 4 pictures, and it takes 160 seconds to take 50 pictures, with an average of about 3.2 seconds per frame.

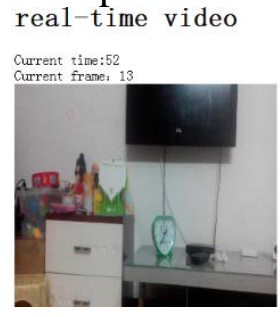

Fig. 3 indoor monitoring screenshot

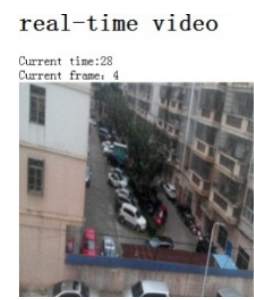

Fig. 4 outdoor monitoring screenshot

The results of experiments showed that the method this method has the advantages of good reusability, expansibility and easy realization. Due to the use of HTTP protocol communication, the system has a low degree of coupling. Due to mobile phone camera image data acquisition based on SL4A, the system will not be affected by the application of android camera, with a high degree of independence. At the same time, SL4A can conveniently integrate the geographic location and other sensor information into the camera image, so the method has good scalability. The script language has the advantages of simple development and powerful function, so it can shorten the development cycle and reduces the difficulty of development. But the method has obvious shortcomings, because video function is done by images of mobile phone camera, it takes time to start, close, and compress the phone camera image service, therefore, the display time of the images is delayed and the images are discontinuous. Another problem about this method is that there is only image data without voice data.

\section{Conclusions}

In this paper, we explore the expansion method of mobile camera for mobile scripting language , design and develop a mobile video monitor system based on SL4A, HTTP and script language. This method can be used in the monitoring occasions that real-time requirement is not high, such as infrastructure of house or small residential areas. The server and client of the system can be implemented in the same scripting language,such as JavaScript, compared with the traditional methods of using Java and scripting language, this method of the paper not only has the advantages of web application, but also greatly reduces the difficulty of development. The next step of the research work is to add voice date, eliminate the time-consuming impact of SL4A and integrate the SL4A sensor information in camera image data. 


\section{Acknowledgment}

This work was financially supported by the Scientific Research Project of Hainan College of Software Technology (Hr201601).

\section{REFERENCES}

[1] Saurabh Saha. Aug 18, 2013. How To Turn Any Old Android Phone Into Remote CCTV Spy Camera/WebCam?[Online].

Available:http://www.techgyd.com/how-to-change-any-old-android-phone-into-cctv-spy-camera-w ebcam/3995/.

[2] ZHANG Yanan, YANG Lu, ZHENG Limin. Remote video surveillance system based on Android mobile phone[J].

Journal of Computer Applications,2013,33(S1):283-286.

[3] YANG Zongxian, ZHANG Chunxia, HU Xiaoya et al. Video Monitoring System based on Android Smart Phone[J]. Computer \& Digital Engineering, 2015,43(08):1536-1539.

[4] damonkohler.(2015,January). Scripting Layer for Android (SL4A) [Online].

Available: https://github.com/damonkohler/sl4a.

[5] P. Ferrill , Pro Android Python with SL4A, Berkeley: APress, 2011.

[6] Y.L. Xing ,et al,. PHP Web and Android Development of entry and Practice, Beijing: Tsinghua University press, 2014 .

[7] Y.L. Xing ,et al,. The Development Technology of Android Script Application, Beijing: Tsinghua University press, 2017 .

[8] irontec. (2015,January). PHP for Android project(PFA)[Online]. Available: http://phpforandroid.net/. 